\title{
Regulation of UHRF1 by microRNA-378 modulates medulloblastoma cell proliferation and apoptosis
}

\author{
ZHEN-YU ZHANG $^{1 *}$, BIN ZHU $^{2 *}$, XIN-WEI ZHAO ${ }^{*}$, YUN-BO ZHAN $^{1}$, JIAN-JI BAO ${ }^{1}$, JIN-QIAO ZHOU ${ }^{1}$, \\ FENG-JIANG ZHANG ${ }^{1}$, BIN YU ${ }^{1}$, JUN LIU ${ }^{1}$, YAN-MIN WANG ${ }^{1}$, YA-HUI BAI ${ }^{1}$, JIN HONG $^{3}$ and XIAN-ZHI LIU ${ }^{1}$ \\ ${ }^{1}$ Department of Neurosurgery, The First Affiliated Hospital of Zhengzhou University, Zhengzhou, Henan 450001; \\ ${ }^{2}$ Queen Mary Institute, Nanchang University, Nanchang, Jiangxi 330000; ${ }^{3}$ Department of Cardiology, \\ The First Affiliated Hospital of Zhengzhou University, \\ Zhengzhou, Henan 450001, P.R. China
}

Received March 15, 2017; Accepted August 5, 2017

DOI: $10.3892 /$ or.2017.5939

\begin{abstract}
A previous study revealed that ubiquitin-like with PHD and RING finger domains 1 (UHRF1) promoted cell proliferation and was a potential biomarker in medulloblastoma (MB). In the present study, we reported that miR-378 inhibited the expression of UHRF1 to affect the proliferation of MB through competitive binding to the same region of its 3'-UTR. We found that the expression of miR-378 was significantly downregulated in MB tissues and inversely correlated with the expression of UHRF1. Western blot analysis revealed that overexpression of miR-378 led to the suppression of UHRF1. Moreover, a dual-luciferase assay demonstrated that miR-378 negatively regulated the activity of target gene UHRF1 by binding to its 3'-UTR. An in vitro assay revealed that overexpression of miR-378 suppressed MB cell proliferation and promoted cell apoptosis. Ectopic expression of UHRF1 rescued miR-378-suppressed cell proliferation and miR-378-promoted cell apoptosis. Collectively, the present study demonstrated that miR-378 could inhibit the proliferation of MB by downregulation of UHRF1 and act as a potential therapeutic target against MB.
\end{abstract}

\section{Introduction}

Medulloblastoma (MB) accounts for approximately one-fifth of all pediatric brain tumors and is one of the most common malignant brain tumors in children (1-3). Due to the progress in radiation therapy and chemotherapy during the past decades, the survival of MB patients has considerably improved $(4,5)$.

Correspondence to: Dr Xian-Zhi Liu, Department of Neurosurgery, The First Affiliated Hospital of Zhengzhou University, 1 Jianshe Dong Road, Zhengzhou, Henan 450001, P.R. China

E-mail: xzliu06@126.com

${ }^{*}$ Contributed equally

Key words: medulloblastoma, microRNA-378, UHRF1, proliferation, apoptosis
Nevertheless, in WHO grade IV central nervous system tumors, the prognosis of MB remains dismal due to rapid recurrence or metastasis in a considerable proportion of the tumors (6). Therefore, it is necessary to gain more insights in the carcinogenesis of MB and explore molecular targets potentially useful for treating the disease.

Ubiquitin-like with PHD and RING finger domains 1 (UHRF1) is comprised of an N-terminal ubiquitylation-like domain, a plant homeodomain (PHD) domain, a RING finger, and a SET- and RING-associated (SRA) domain, which plays a major role in DNA methylation and cell proliferation $(7,8)$. The SRA domain of UHRF1 recognizes hemi-methylated DNA, recruits DNA methyltransferase 1 (DNMT1) and maintains DNA-methylation patterns (9). In the past few years, UHRF1 was revealed to be aberrantly upregulated and functioned as an oncogene in various types of cancers, including laryngeal squamous cell carcinoma (10), non-small cell lung (11), breast (12) and gastric cancer (13), hepatocellular carcinoma (14), and colorectal (15), bladder (16) and prostate cancer (17). Most recently, we demonstrated that UHRF1 was overexpressed in MB tissues and was an independent prognostic factor. We further revealed that UHRF1 promoted the proliferation and progression of MB cells, indicating that UHRF1 could be a potential therapeutic target for MB (18).

MicroRNAs (miRNAs), a class of $\sim 19-25$ nt non-coding RNAs, are evolutionarily conserved, and serve as endogenous regulators of gene expression. They also play a vital role in carcinogenesis. miRNAs could cause either mRNA degradation or inhibition of protein translation via direct interaction with the 3'-UTR of target mRNA $(19,20)$. A growing body of compelling data has revealed that the malfunction of certain miRNAs is involved in a series of tumorigenic processes including cell proliferation, apoptosis, metastasis and angiogenesis (21). Recent studies revealed that dysregulation of miR-378 was shown related to tumorigenesis and cancer progression. Notably, miR-378 was reported as an onco-miR in nasopharyngeal carcinoma (22), but as a tumor suppressor microRNA in colorectal (23) and prostate cancer (24), and glioma (25). Nevertheless, to the best of our knowledge, the molecular mechanism of miR-378 in the regulation of $\mathrm{MB}$ is unknown. 
Playing a vital part in connection to DNA methylation, UHRF1 was implicated in MB carcinogenesis in our previous study (18). However, little is known about the factors that modulated UHRF1 expression. In the present study, we found that miR-378 was downregulated in MB specimens and was inversely associated with UHRF1 expression. We further predicted and confirmed that miR-378 modulated the expression of UHRF1, and exerted a tumor-suppressive function in MB proliferation.

\section{Materials and methods}

Patients, tissue specimens. Fresh frozen tissues of 19 patients with MB and 9 cases of normal cerebellum (collected in brain trauma surgery) were gathered at The First Affiliated Hospital of Zhengzhou University between January 2015 and September 2016. Informed consent was obtained from all individual participants included in the study and all procedures performed involving human participants were in accordance with the ethical standards of the Institutional Research Committee of The First Affiliated Hospital of Zhengzhou University.

Cell culture and transfection. DAOY and HEK 293T cell lines were purchased from American Type Culture Collection (ATCC, Manassas, VA, USA). ONS-76 cell line was obtained from Japanese Collection of Research Bioresources Cell Bank (JCRB Cell Bank, Osaka, Japan). ONS-76 cells were cultured in RPMI-1640 medium (Thermo Scientific HyClone, Beijing, China) and DAOY cells were maintained in MEM- $\alpha$ medium (Life Technologies, Carlsbad, CA, USA). The transfection was performed using Lipofectamine 2000 reagent (Invitrogen, Carlsbad, CA, USA) according to the manufacturer's instructions. miR-378 was cloned into the CMV-miRNA-PGKpuromycin vector (Genomeditech, Shanghai, China). UHRF1 cDNA was cloned into pLVX-Neo-IRES-ZsGreen1 (Genomeditech). Puromycin was used to establish the miR-378 stably transfected cell lines. Fluorescence was detected for selection of clones with stable UHRF1 expression. Stably transfected clones were validated by quantitative real-time polymerase chain reaction (qRT-PCR) and immunoblotting. Transfected cells were then used for Cell Counting Kit-8 (CCK-8) and clonogenic assays, and flow cytometry.

Real-time quantitative PCR. Small RNAs were isolated from tissue samples with the mirVana miRNA Isolation kit (Ambion, Austin, TX, USA) following the manufacturer's instructions. The total RNA of the fresh tissues, ONS-76 and DAOY cells was extracted using TRIzol reagent (Invitrogen) and reversely transcribed with RT Primer Mix and PrimeScript RT Enzyme Mix 1 (Takara, Shiga, Japan). The mRNA level was quantified with SYBR Premix Ex Taq (Takara). Human-U6 RNA was chosen as an endogenous control. The PCR reaction was conducted with a real-time PCR system (Roche Diagnostics, Penzberg, Germany). Conditions for amplification were $94^{\circ} \mathrm{C}$ for $2 \mathrm{~min}$ followed by 40 cycles of $94^{\circ} \mathrm{C}$ for $25 \mathrm{sec}, 61^{\circ} \mathrm{C}$ for $31 \mathrm{sec}$. The fold change was calculated using the $2^{-\Delta \Delta \mathrm{Ct}}$ method. The levels of miR-378 in normal brain tissues were normalized to an arbitrary value of 1 . The primers used are listed in Table I.

Western blot analysis. Total protein was extracted from fresh tissues, ONS-76 and DAOY cells using cell lysates. Gene
Table I. Primers used in quantitative real-time PCR.

\begin{tabular}{lll}
\hline Primer name & \multicolumn{2}{c}{ Sequence (5'-3') } \\
\hline Pre-miR-378 & F & CGACGCGTCGGGCTGCGAGGAGTGAGCG \\
& R & CCATCGATGGGAGTTCAAATGGCTTGCTCC \\
U6 & F & TGCGGGTGCTCGCTTCGGCAGC \\
& R & CCAGTGCAGGGTCCGAGGT \\
UHRF1 & F & CCACATCGTCCTCACAGC \\
& R & GGTCCACATCATCCTCATAGC \\
GAPDH & F & TGATGACATCAAGAAGGTGGTGAAG \\
& R & TCCTTGGAGGCCATGTGGGCCAT \\
\hline
\end{tabular}

F, forward; R, reverse; UHRF1, ubiquitin-like with PHD and RING finger domains 1; GAPDH, glyceraldehyde-3-phosphate dehydrogenase.

expression was normalized with glyceraldehyde-3-phosphate dehydrogenase (GAPDH). Fifty micrograms of samples were first electrophoresed on a $10 \%$ SDS-polyacrylamide gels, transferred to nitrocellulose filter membranes, and incubated in blocking solution consisting of 5\% non-fat milk in Tris-buffered saline with Tween-20 (TBST) at room temperature for $1 \mathrm{~h}$. Subsequently, the membranes were immunoblotted with UHRF1 (1:250 dilution; BD Biosciences, San Jose, CA, USA), cleaved PARP (1:1,000 dilution), cleaved caspase-3 (1:1,000 dilution) and GAPDH antibodies (1:5,000 dilution) [all from Cell Signaling Technology (CST) Beverly, MA, USA], overnight at $4^{\circ} \mathrm{C}$. Subsequently, the membranes were washed and incubated with appropriate HRP-conjugated secondary IgG antibodies (Dako, Glostrup, Denmark). The protein bands were visualized using ECL Western Blotting Substrate (Pierce; Thermo Fisher Scientific, Inc., Waltham, MA, USA).

Target prediction and dual-luciferase reporter assay. Three miRNA target prediction algorithms were used (miRWalk 2.0, TargetScan and microrna.org). For the dual-luciferase report assay, the wild-type (WT) reporter construct pmirGLO/UHRF1-3'-UTR and the mutant (MUT) reporter construct pmirGLO/UHRF1-3'-UTR MUT were cloned. The site of perfect complementarity to miR-378 was mutated using site-directed mutagenesis PCR. Subsequently, 293 cells were co-transfected with miR-378 in a 48-well plate followed by the pmirGLO/UHRF1-3'-UTR reporter vector or the pmirGLO/ UHRF1-3'-UTR MUT vector. Firefly luciferase activity for each sample was normalized to Renilla luciferase activity and was assessed at $48 \mathrm{~h}$ after transfection.

CCK-8 assay. Cell viability was assessed using a CCK-8 assay. Stably transfected ONS-76 and DAOY cells were seeded at a density of $10^{3}$ cells/well into 96-well culture plates and incubated for 24, 48, 72 and $96 \mathrm{~h}$. For the CCK-8 assay, the cells were further incubated with $10 \mu \mathrm{l} \mathrm{CCK-8} \mathrm{(Sigma,} \mathrm{Santa} \mathrm{Clara,}$ CA, USA) for $4 \mathrm{~h}$. The absorbance was assessed at $450 \mathrm{~nm}$.

Clonogenic assay. A flat plate clone formation assay was used to investigate cell clonogenic ability. Cells in the logarithmic growth phase were digested into a single-cell suspension using a trypsin-EDTA solution, and $2 \mathrm{ml}$ of cell suspension 

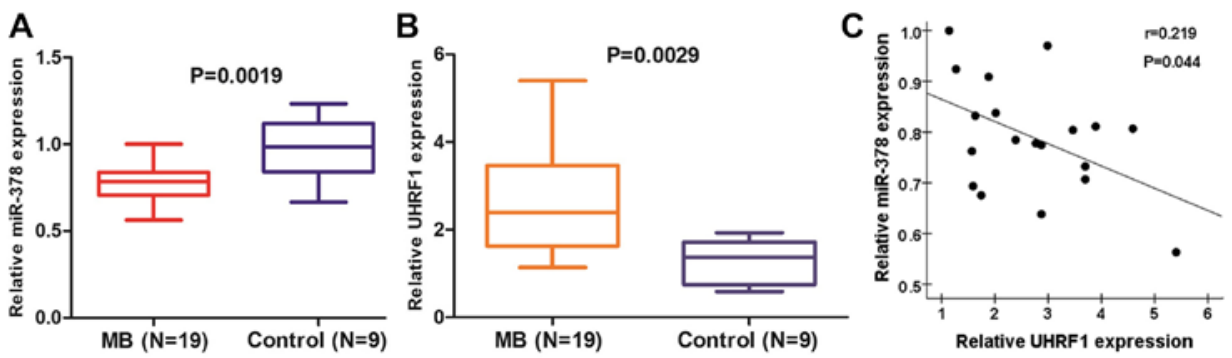

Figure 1. miR-378 is downregulated in MB tissues and inversely correlated with UHRF1. (A) The endogenous level of miR-378 in MB and normal cerebellum tissues by real-time qRT PCR. The expression of miR-378 was normalized to U6 small nuclear RNA ( $\mathrm{P}=0.0019)$. (B) The mRNA level of UHRF1 in MB and normal cerebellum tissues by real-time qRT PCR. The expression of UHRF1 mRNA was normalized to GAPDH (P=0.0029). (C) Inverse correlation between miR-378 and UHRF1 mRNA levels in MB specimens ( $\mathrm{P}=0.044)$.

A

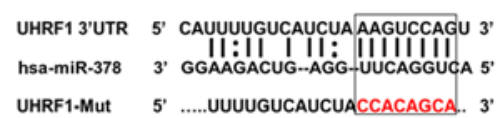

D

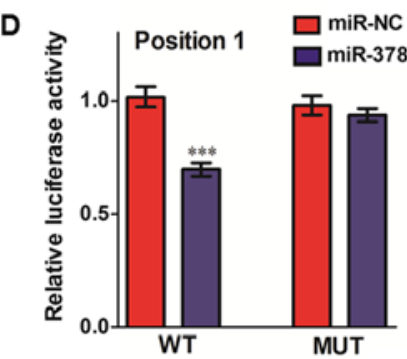

G

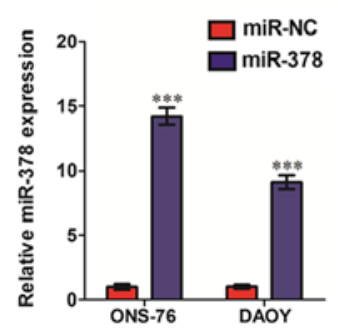

B

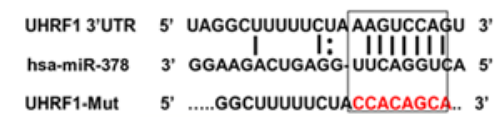

E

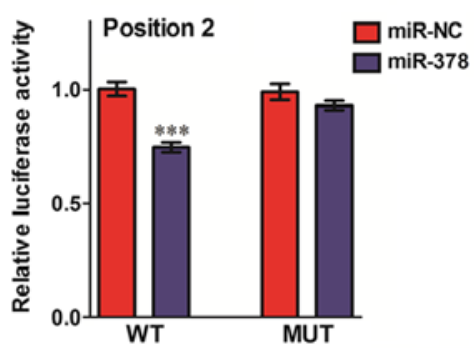

H

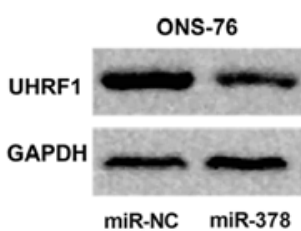

c

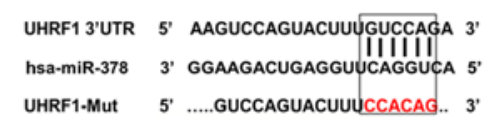

$\mathbf{F}$
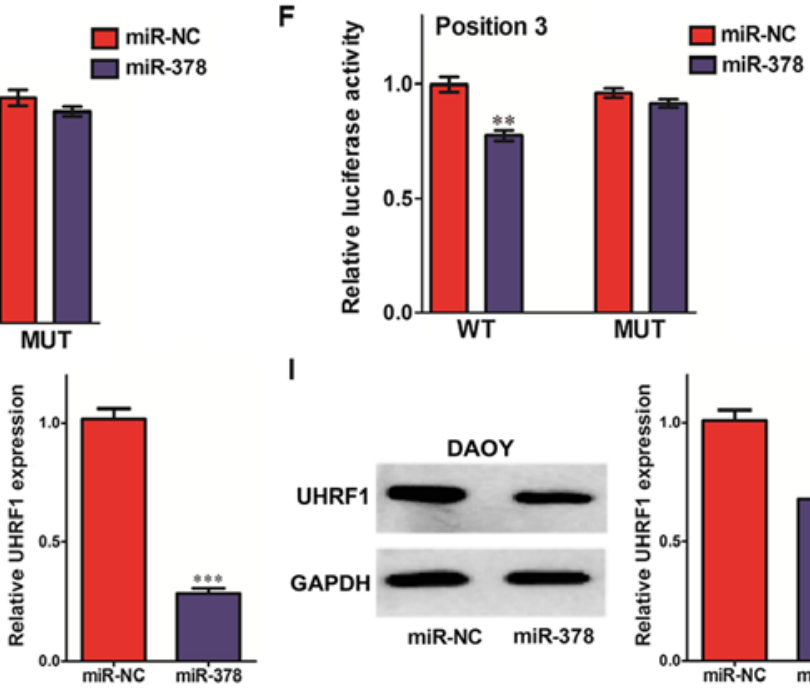

I

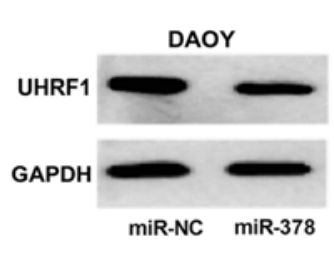

Figure 2. miR-378 directly downregulates the expression of UHRF1 in MB. (A-C) Three predicted binding sites for miR-378 in the 3'-UTR of UHRF1 and corresponding mutations in the binding sites are shown. (D-F) Luciferase constructs of wild-type (WT) or mutant (MT) UHRF1 were transfected into 293 cells with miR-378/miR-NC. The luciferase activity was detected after $48 \mathrm{~h}$. The ratio was normalized to control luciferase activity. miR-378 decreased the intensity of the luciferase-UHRF1 3'-UTR reporter vector, while the MT luciferase-UHRF1 3'-UTR failed to decrease the luciferase intensity (position 1 $\mathrm{P}<0.001$; position $2 \mathrm{P}<0.001$; position $3 \mathrm{P}<0.01)$. (G) qRT PCR demonstrated that the level of miR-378 was significantly increased in ONS-76 (P<0.001) and DAOY $(\mathrm{P}<0.001)$ cells after transfection with miR-378. (H and I) Overexpression of miR-378 significantly inhibited the expression of UHRF1 in ONS-76 $(\mathrm{P}<0.001)$ and DAOY $(\mathrm{P}<0.05)$ cells; ${ }^{*} \mathrm{P}<0.05,{ }^{* *} \mathrm{P}<0.01,{ }^{* * * *} \mathrm{P}<0.001$.

was then seeded onto a 6 -well cell culture plate at a density of 1,000 cells $/ \mathrm{ml}$. The cells were cultured at $37^{\circ} \mathrm{C}$ in a well-humidified $95 \%$ air $/ 5 \% \mathrm{CO}_{2}$ incubator for 2 weeks, and the colony formation was photographed and counted.

Flow cytometry for cell apoptosis. The apoptotic ratios of cells were determined using the Annexin V/7-ADD apoptosis detection kit (Roche, Basel, Switzerland). Forty-eight hours after transfection, the cells were harvested and washed twice with phosphate-buffered saline (PBS) buffer, suspended with binding buffer, incubated with Annexin V-R-PE for $20 \mathrm{~min}$ in a dark ice bath, and then with 7-AAD before analysis on a BD FACSCalibur (BD Biosciences).

Statistical analysis. All in vitro experiments were repeated at least 3 times. The numerical data were documented as the mean \pm standard deviation (SD). The Student's t-test or one-way analyses of variance were employed to compare the differences in numerical variables between different groups. Statistical significance was defined as a P-value $<0.05$. Data statistics were performed using software GraphPad Prism 5 (GraphPad Software, Inc., La Jolla, CA, USA) and IBM SPSS Statistics 19 (IBM Corp., Armonk, NY, USA).

\section{Results}

miR-378 is downregulated in MB tissues and inversely correlated with UHRF1. To evaluate the expression of miR-378 in $\mathrm{MB}$, we first quantitatively detected the levels of reverse transcription-PCR in $19 \mathrm{MB}$ and 9 normal cerebellum tissues. The results demonstrated that miR-378 was considerably decreased in MB compared with normal cerebellum (Fig. 1A). Meanwhile, the expression of UHRF1 was revealed to be significantly upregulated in MB tissues compared with normal 
A

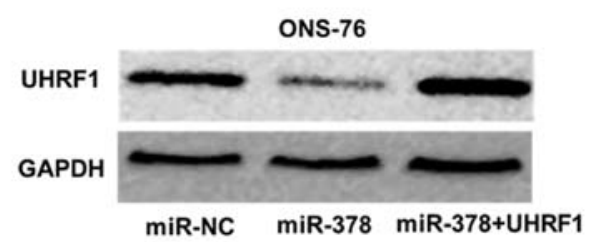

C

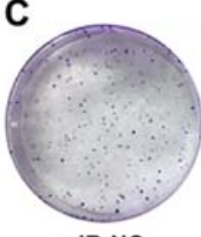

miR-NC

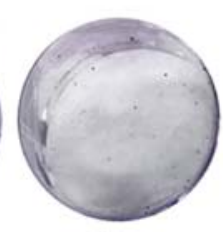

miR-378

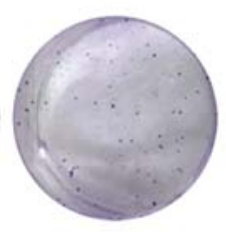

miR-378+UHRF1

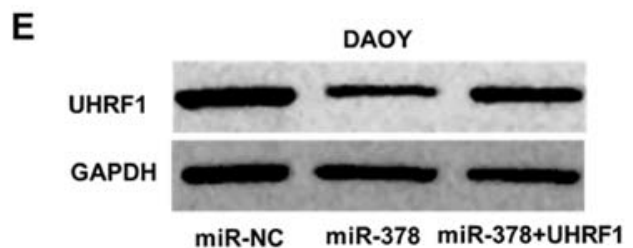

G
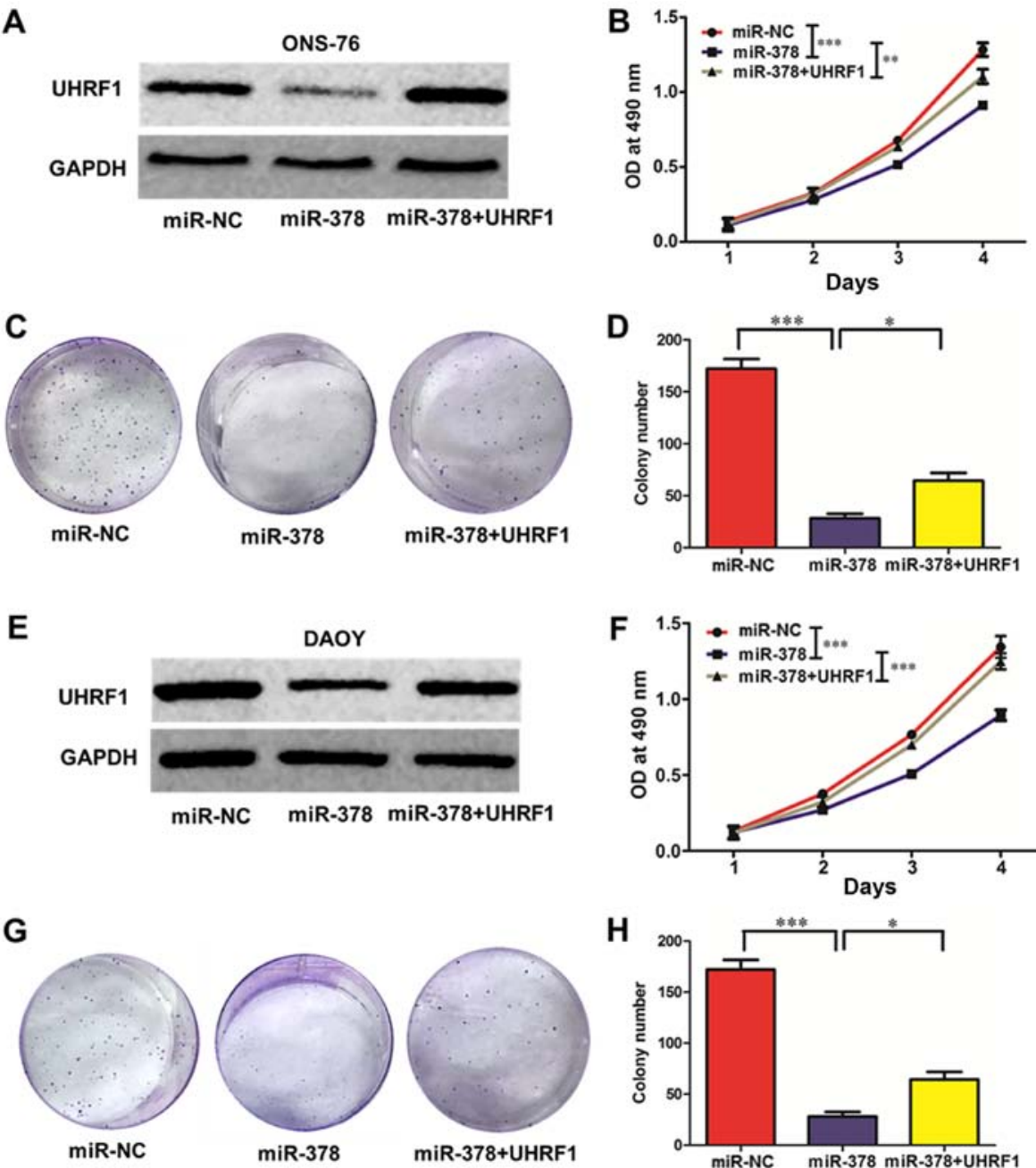

D
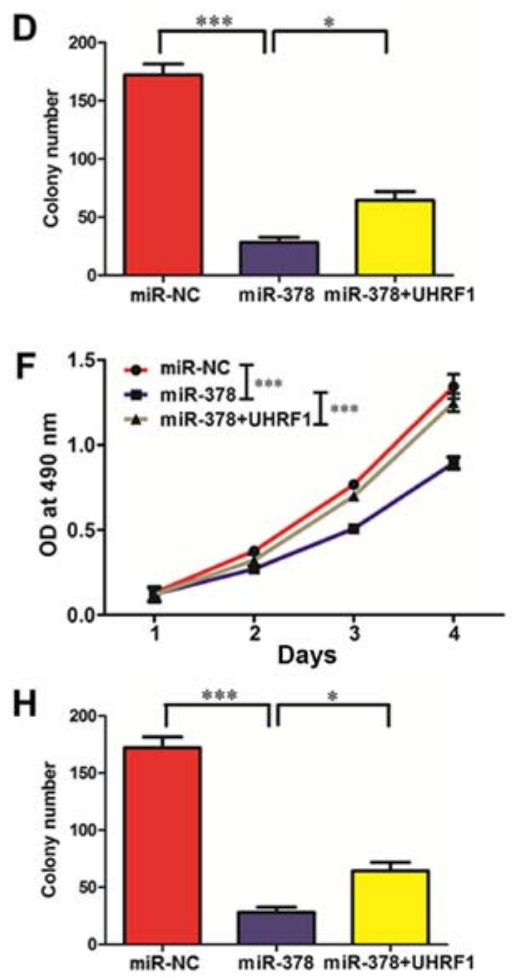

Figure 3. miR-378 regulates ONS-76 and DAOY cell viability and clonogenic ability. (A and E) The protein expression of UHRF1 was assessed by western blot analysis. ONS-76 and DAOY cells were transfected with control (miR-NC), miR-378, and miR-378 along with UHRF1. miR-378 suppressed UHRF1 protein expression compared with the control group, while UHRF1 rescued the protein level of UHRF1 compared with that of the miR-378 group. (B and F) Cell viability was detected by CCK-8 assays. After transfection with miR-NC, miR-378 and miR-378 along with UHRF1 in (B) ONS-76 and (F) DAOY cells, the CCK-8 assay was used to determine the relative cell viability at 1, 2, 3 and 4 days. (C, D, G and H) Cell clonogenic ability was evaluated by colony formation assay. (C and D) ONS-76 and (G and H) DAOY cells that were transfected with miR-NC, miR-378 and miR-378 along with UHRF1 were seeded into 12-well plates. On the 12th day after seeding, the number of colonies was counted; ${ }^{*} \mathrm{P}<0.05,{ }^{* * *} \mathrm{P}<0.01,{ }^{* * * *} \mathrm{P}<0.001$.

cerebellum tissues (Fig. 1B). In combination with the two data, an inverse correlation between the mRNA levels of miR-378 and UHRF1 in MB tissues was observed (Fig. 1C).

miR-378 is a potent miRNA regulator of UHRF1 expression. Three miRNA target prediction algorithms including miRWalk 2.0, Targetscan and microrna.org were used to search for potential miRNA binding sites with the 3'-UTR region of UHRF1. Due to the higher prediction score (data not shown), we chose miR-378 for further study. As shown in Fig. 2A-C, the 3 '-UTR of UHRF1 mRNA contained a putative binding site for miR-378. We then cloned the human UHRF1 3'-UTR sequence and inserted it into a luciferase reporter plasmid and co-transfected it with miR-378 into 293 cells. The luciferase reporter assays demonstrated that miR-378 markedly inhibited the activity of firefly luciferase that contained the WT but not the MUT 3'-UTR of UHRF1 (Fig. 2D-F). Moreover, we further investigated whether miR-378 could modulate the endogenous expression of UHRF1 in MB cells. After transfection of mature miR-378 by ectopic plasmids in MB cells, the expression levels of miR-378 were substantially increased, $\sim 14$-fold in ONS-76 cells and 9-fold in DAOY cells, respectively, compared to the miR-NC control (Fig. 2G). Consistently with previous luciferase reporter assays, overexpression of miR-378 significantly inhibited UHRF1 expression in ONS-76 and DAOY cells (Fig. 2H and I). These data indicated that miR-378 negatively regulated the expression of UHRF1 by directly binding to its 3 '-UTR.

Overexpression of miR-378 suppresses the proliferation of $M B$ cells and restoration of UHRF1 counteracts the effect of miR-378 in part. To better understand how miR-378 influences the proliferation ability of MB cells, CCK- 8 and colony formation assays were conducted in ONS-76 and DAOY cells. In the meantime, to confirm whether the effect of miR-378 on MB cells is mediated by its regulatory role on UHRF1 expression, a rescue assay was performed. Forced overexpression of miR-378 resulted in a marked decrease in the expression of UHRF1, and this decrease was rescued by transfection of UHRF1 (Fig. 3A and E). A CCK-8 assay 
A

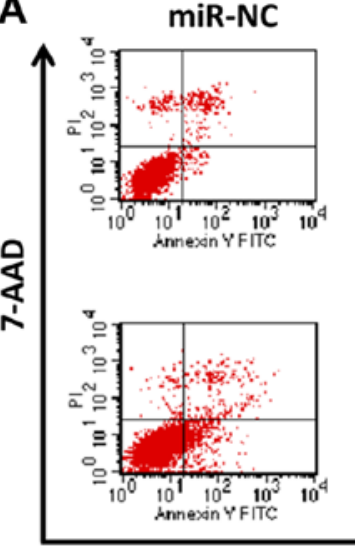

B

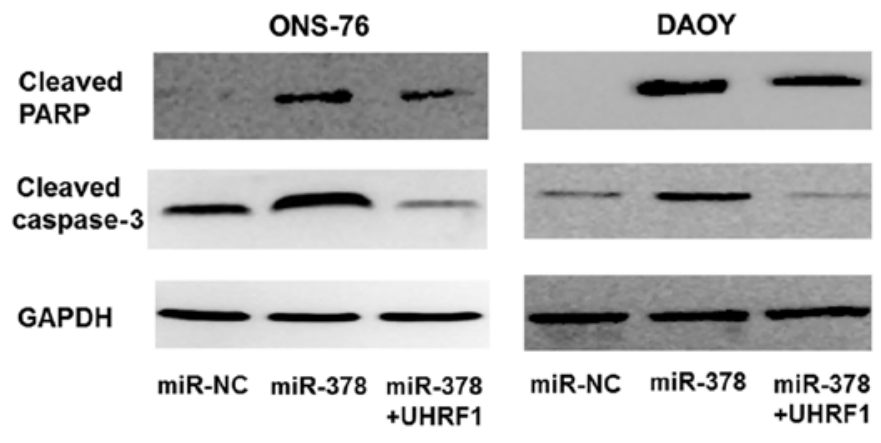

$\operatorname{miR}-378$
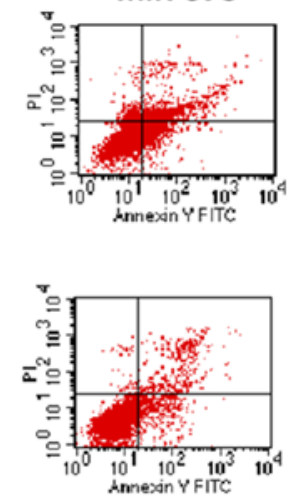

Annexin V
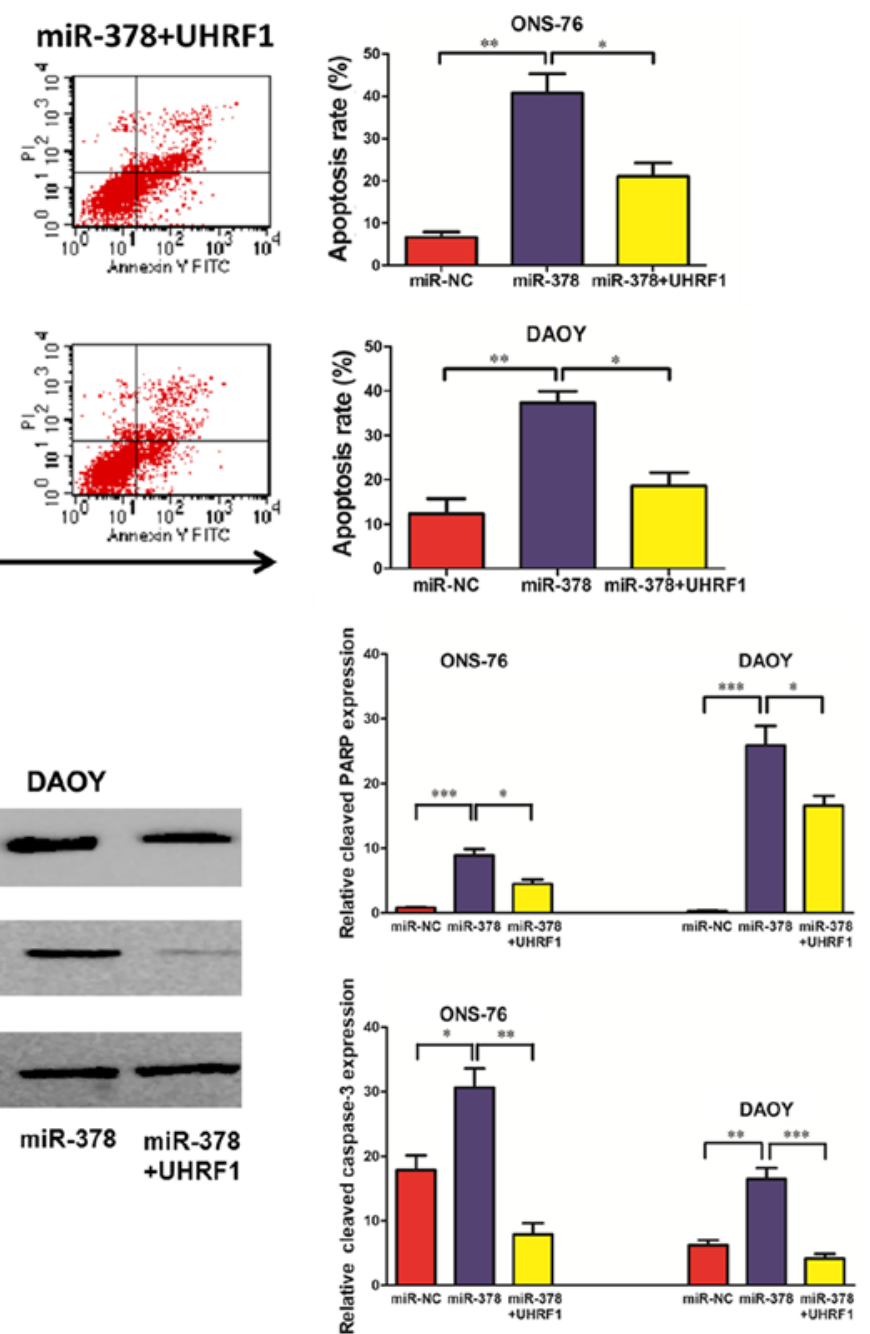

Figure 4. miR-378 regulates ONS-76 and DAOY cell apoptosis and the related mechanism. (A) The incidence of apoptotic cells was studied by flow cytometry. In ONS-76 and DAOY cells, the percentage of total apoptotic cells increased in response to miR-378 upregulation compared with the control group, and ectopic overexpression of UHRF1 partly reversed this tendency. The cells were stained with Annexin V-fluorescein in isothiocyanate and counterstained with 7-ADD. (B) Western blotting revealed that forced expression of miR-378 markedly increased the protein levels of cleaved PARP and cleaved caspase-3 in ONS-76 and DAOY cells, and ectopic expression of UHRF1 partly suppressed the expression of the two apoptosis-related proteins; ${ }^{*} \mathrm{P}<0.05,{ }^{* *} \mathrm{P}<0.01,{ }^{* * *} \mathrm{P}<0.001$.

revealed that upregulation of miR-378 substantially suppressed the viability of ONS-76 and DAOY cells, and ectopic expression of UHRF1 partly rescued the inhibition of viability (ONS-76 MB cells, Fig. 3B; DAOY MB cells, Fig. 3F). Additionally, the plate colony formation assay revealed that the colony formation ability was markedly decreased in miR-378 overexpressed MB cells, and restoration of UHRF1 partly increased the formation ability (ONS-76 MB cells, Fig. 3C and D; DAOY MB cells, Fig. 3G and $\mathrm{H})$.

Overexpression of miR-378 promotes apoptosis and restoration of UHRF1 counteracts the effect of miR-378 in part. Fluorescence-activated cell sorting (FACS) analysis was used to investigate the effects of miR-378 on MB cell apoptosis. The results demonstrated that enforced expression of miR-378 led to significant cancer cell apoptosis. The percentage of total apoptotic cells was markedly increased in response to miR-378 upregulation compared with the control group in ONS-76 and DAOY cells. Moreover, ectopic overexpression of UHRF1 reversed this tendency (Fig. 4A). We subsequently investigated the expression of two putative apoptosis-related proteins PARP and activated caspase-3 in ONS-76 and DAOY cells. Western blotting revealed that upregulation of miR-378 markedly increased the expression of cleaved PARP and cleaved caspase-3 in ONS-76 and DAOY cells, and ectopic expression of UHRF1 partly inhibited the expression of the two apoptosis-related proteins (Fig. 4B).

\section{Discussion}

As a putative oncogene, UHRF1 has been demonstrated to be involved in the process of cancer cell proliferation, invasion and apoptosis. UHRF1 has also been revealed to be upregulated in various types of cancer, and its expression was associated with poor clinical outcomes (9-17). In our previous study (18), UHRF1 expression was not detected in normal cerebellum tissues but was detected in the majority of MB tissues. In univariate and multivariate survival analysis, the expression level of UHRF1 was identified as an independent prognostic factor influencing overall survival (OS) and progression-free survival (PFS) of MB patients. Furthermore, downregulation of UHRF1 by RNAi inhibited the proliferation 
and clonogenic ability of MB cell lines with cell cycle arrest at the $\mathrm{G} 1 / \mathrm{G} 2$ phase. These data for the first time revealed the important roles of UHRF1 in MB tumorigenesis and progression and suggested that UHRF1 may be a potential therapeutic target for MB.

Recently, studies have implicated miRNAs as important modulators in MB cell proliferation and migration. For instance, miR-218 functions as a tumor suppressor by inhibiting several phenotype-associated genes in MB (26). miR-124, miR-219 and miR-383 were reported to suppress cell proliferation, migration and invasion of MB cells (27-29). miR-378 was reported to function as a tumor suppressor microRNA in several types of cancer including glioma (23-25). Nonetheless, whether and how miR-378 plays a role in MB development and progression remains unexplored.

Based on our previous study, we used various miRNA target prediction algorithms and predicted miR-378 as one of the most possible miRNAs that interact with the 3'-UTR of UHRF1. Several previous studies had revealed that the expression of miRNA-378 was decreased in certain tumors $(30,31)$. It has been reported that miR-378 was significantly downregulated in colorectal cancer tissues and forced expression of miR-378 inhibited tumor cell viability and invasion (30). Fei and $\mathrm{Wu}$ demonstrated that the expression of miR-378 was decreased in gastric cancer tissues and cell lines, and delivery of miR-378 could hinder cell proliferation and induce cell apoptosis (31). In the present study, we used a luciferase reporter assay to validate the interaction between miRNA-378 and UHRF1, and the results revealed that miR-378 negatively regulated the expression of UHRF1. Subsequent transfection of miR-378 in MB cell lines resulted in the inhibition of proliferation, colony formation ability and promotion of cell apoptosis. A restoration assay demonstrated that transfection of UHRF1 could partly counteract the effect of miR-378, indicating that miR-378 mediated the biological behavior of $\mathrm{MB}$ cells, at least partly via regulation of UHRF1.

Even though the present study first demonstrated that miR-378 negatively regulates UHRF1, it has certain limitations. For instance, due to the limited sample size of MB and normal cerebellum tissues, the association between the expression of miR-378 in tumors and controls was preliminary, and the sample size needs to be increased for more compelling evidence. Additionally, extensive biological and functional characterization of miR-378 and UHRF1 are needed to better clarify the regulation of miR-378 on UHRF1 in MB carcinogenesis and progression in vitro and in vivo.

In conclusion, our findings revealed that UHRF1, a cancer gene in MB demonstrated by our previous study, is in a modulation axis downstream of miR-378. In addition, our results shed light on the roles of miR-378, which negatively regulated UHRF1, in proliferation and apoptosis of MB cells. Although more detailed experimental and clinical investigations to better clarify the mechanism of miR-378 and its effects on MB patients are warranted, the present study shed light on the promotion of a novel targeted therapy for MB treatment.

\section{Acknowledgements}

The authors are thankful for the financial support of the Youth Innovation Fund of The First Affiliated Hospital of
Zhengzhou University to Y.-M.W. and to Y.-H.B., the Science and Technology Program of Henan Province to J.-Q.Z. (172102310648), and the Medical Science and Technology Program of Henan Province to J.-Q.Z. (201602058).

\section{References}

1. Gottardo NG, Hansford JR, McGlade JP, Alvaro F, Ashley DM, Bailey S, Baker DL, Bourdeaut F, Cho YJ, Clay M, et al: Medulloblastoma Down Under 2013: A report from the third annual meeting of the International Medulloblastoma Working Group. Acta Neuropathol 127: 189-201, 2014.

2. Kool M, Korshunov A, Remke M, Jones DT, Schlanstein M, Northcott PA, Cho YJ, Koster J, Schouten-van Meeteren A, van Vuurden D, et al: Molecular subgroups of medulloblastoma: An international meta-analysis of transcriptome, genetic aberrations, and clinical data of WNT, SHH, Group 3, and Group 4 medulloblastomas. Acta Neuropathol 123: 473-484, 2012.

3. Packer RJ, Cogen P, Vezina G and Rorke LB: Medulloblastoma: Clinical and biologic aspects. Neuro-oncol 1: 232-250, 1999.

4. Packer RJ, Sutton LN, Goldwein JW, Perilongo G, Bunin G, Ryan J, Cohen BH, D'Angio G, Kramer ED, Zimmerman RA, et al: Improved survival with the use of adjuvant chemotherapy in the treatment of medulloblastoma. J Neurosurg 74: 433-440, 1991.

5. del Charco JO, Bolek TW, McCollough WM, Maria BL, Kedar A, Braylan RC, Mickle JP, Buatti JM, Mendenhall NP and Marcus RB Jr: Medulloblastoma: Time-dose relationship based on a 30-year review. Int J Radiat Oncol Biol Phys 42: 147-154, 1998.

6. Northcott PA, Korshunov A, Witt H, Hielscher T, Eberhart CG, Mack S, Bouffet E, Clifford SC, Hawkins CE, French P, et al: Medulloblastoma comprises four distinct molecular variants. J Clin Oncol 29: 1408-1414, 2011.

7. Bronner C, Achour M, Arima Y, Chataigneau T, Saya H and Schini-Kerth VB: The UHRF family: Oncogenes that are drugable targets for cancer therapy in the near future? Pharmacol Ther 115: 419-434, 2007.

8. Muto M, Kanari Y, Kubo E, Takabe T, Kurihara T, Fujimori A and Tatsumi K: Targeted disruption of $N p 95$ gene renders murine embryonic stem cells hypersensitive to DNA damaging agents and DNA replication blocks. J Biol Chem 277: 34549-34555, 2002.

9. Mudbhary R, Hoshida Y, Chernyavskaya Y, Jacob V, Villanueva A, Fiel MI, Chen X, Kojima K, Thung S, Bronson RT, et al: UHRF1 overexpression drives DNA hypomethylation and hepatocellular carcinoma. Cancer Cell 25: 196-209, 2014.

10. Pi JT, Lin Y, Quan Q, Chen LL, Jiang LZ, Chi W and Chen HY: Overexpression of UHRF1 is significantly associated with poor prognosis in laryngeal squamous cell carcinoma. Med Oncol 30: $613,2013$.

11. Daskalos A, Oleksiewicz U, Filia A, Nikolaidis G, Xinarianos G, Gosney JR, Malliri A, Field JK and Liloglou T: UHRF1-mediated tumor suppressor gene inactivation in nonsmall cell lung cancer. Cancer 117: 1027-1037, 2011.

12. Geng Y, Gao Y, Ju H and Yan F: Diagnostic and prognostic value of plasma and tissue ubiquitin-like, containing PHD and RING finger domains 1 in breast cancer patients. Cancer Sci 104: 194-199, 2013.

13. Zhou L, Zhao X, Han Y, Lu Y, Shang Y, Liu C, Li T, Jin Z, Fan D and $\mathrm{Wu}$ K: Regulation of UHRF1 by miR-146a/b modulates gastric cancer invasion and metastasis. FASEB J 27: 4929-4939, 2013.

14. Liang D, Xue H, Yu Y, Lv F, You W and Zhang B: Elevated expression of UHRF1 predicts unfavorable prognosis for patients with hepatocellular carcinoma. Int J Clin Exp Pathol 8: 9416-9421, 2015.

15. Wang F, Yang YZ, Shi CZ, Zhang P, Moyer MP, Zhang HZ, Zou Y and Qin HL: UHRF1 promotes cell growth and metastasis through repression of p16 $6^{\text {ink4a }}$ in colorectal cancer. Ann Surg Oncol 19: 2753-2762, 2012.

16. Yang GL, Zhang LH, Bo JJ, Chen HG, Cao M, Liu DM and Huang YR: UHRF1 is associated with tumor recurrence in non-muscle-invasive bladder cancer. Med Oncol 29: 842-847, 2012.

17. Jazirehi AR, Arle D and Wenn PB: UHRF1: A master regulator in prostate cancer. Epigenomics 4: 251-252, 2012. 
18. Zhang ZY, Cai JJ, Hong J, Li KK, Ping Z, Wang Y, Ng HK, Yao Y and Mao Y: Clinicopathological analysis of UHRF1 expression in medulloblastoma tissues and its regulation on tumor cell proliferation. Med Oncol 33: 99, 2016.

19. Bartel DP: MicroRNAs: Genomics, biogenesis, mechanism, and function. Cell 116: 281-297, 2004.

20. Krol J, Loedige I and Filipowicz W: The widespread regulation of microRNA biogenesis, function and decay. Nat Rev Genet 11: 597-610, 2010.

21. Sato F, Tsuchiya S, Meltzer SJ and Shimizu K: MicroRNAs and epigenetics. FEBS J 278: 1598-1609, 2011.

22. Yu BL, Peng XH, Zhao FP, Liu X, Lu J, Wang L, Li G, Chen HH and Li XP: MicroRNA-378 functions as an onco-miR in nasopharyngeal carcinoma by repressing TOB2 expression. Int $J$ Oncol 44: 1215-1222, 2014.

23. Zhang GJ, Zhou H, Xiao HX, Li Y and Zhou T: MiR-378 is an independent prognostic factor and inhibits cell growth and invasion in colorectal cancer. BMC Cancer 14: 109, 2014.

24. Chen QG, Zhou W, Han T, Du SQ, Li ZH, Zhang Z, Shan GY and Kong CZ: MiR-378 suppresses prostate cancer cell growth through downregulation of MAPK1 in vitro and in vivo. Tumour Biol 37: 2095-2103, 2016.

25. Li B, Wang Y, Li S, He H, Sun F, Wang C, Lu Y, Wang X and Tao B: Decreased expression of miR-378 correlates with tumor invasiveness and poor prognosis of patients with glioma. Int $J$ Clin Exp Pathol 8: 7016-7021, 2015.
26. Venkataraman S, Birks DK, Balakrishnan I, Alimova I, Harris PS, Patel PR, Handler MH, Dubuc A, Taylor MD, Foreman NK, et al: MicroRNA 218 acts as a tumor suppressor by targeting multiple cancer phenotype-associated genes in medulloblastoma. J Biol Chem 288: 1918-1928, 2013.

27. Li KK, Pang JC, Lau KM, Zhou L, Mao Y, Wang Y, Poon WS and $\mathrm{Ng} \mathrm{HK}$ : MiR-383 is downregulated in medulloblastoma and targets peroxiredoxin 3 (PRDX3). Brain Pathol 23: 413-425, 2013.

28. Shi JA, Lu DL, Huang $X$ and Tan W: miR-219 inhibits the proliferation, migration and invasion of medulloblastoma cells by targeting CD164. Int J Mol Med 34: 237-243, 2014.

29. Silber J, Hashizume R, Felix T, Hariono S, Yu M, Berger MS Huse JT, VandenBerg SR, James CD, Hodgson JG, et al: Expression of miR-124 inhibits growth of medulloblastoma cells. Neuro Oncol 15: 83-90, 2013.

30. Zhang GJ, Zhou H, Xiao HX, Li Y and Zhou T: MiR-378 is an independent prognostic factor and inhibits cell growth and invasion in colorectal cancer. BMC Cancer 14: 109, 2014.

31. Fei B and Wu H: MiR-378 inhibits progression of human gastric cancer MGC-803 cells by targeting MAPK1 in vitro. Oncol Res 20: 557-564, 2012. 